\title{
Early-stage ovarian cancer management: still a challenge
}

\author{
Antonio González Martín
}

$\mathrm{O}$ varian cancer is the leading cause of death due to gynaecological tumours in Western countries [1]. The main reason for this is related to the fact that a great majority of patients are diagnosed with advanced disease. Only $20 \%$ of patients are diagnosed in an early stage, when the probability of cure is significantly higher [2]. In this issue of Clinical and Translational Oncology, a large retrospective study of 158 patients with early-stage ovarian cancer treated at a single institution over a period of 22 years is presented by García-Sáez et al [3]. During this long period, several trials have provided the mainstay of management of this disease.

The cornerstone for the treatment of early-stage ovarian carcinoma remains surgery [4]. Optimal staging surgery should be done by a specialised surgeon and must include a vertical supra- and infraumbilical midline incision in order to ensure access to the upper abdomen, which cannot be explored adequately using a transverse lower abdominal or Pfannenstiel incision. Once the abdomen has been opened, the liquid is collected or exfoliative cytology is completed by means of washings. A meticulous inspection of the peritoneal cavity is performed, as well as a thorough evaluation of the abdomen and pelvis. Next, the pathology within the pelvis should be examined. Any adnexal tumour should be removed without rupture as any rupture or release of malignant cells within the peritoneal cavity can result in aggravating the patient's stage, changing the prognosis. A lesion with malignancy confirmed by a frozen section analysis requires a total abdominal hysterectomy as well as a bilateral salpingo-ophorectomy. An omentectomy, a pelvic and para-aortic lymph node inspection and multiple peritoneal biopsies are performed. When carrying out a staging performance of this type, it is common to find that

\footnotetext{
A. González Martín (凶)

Medical Oncology Department

Centro Oncológico MD Anderson International

C/ Arturo Soria, 270

ES-28033 Madrid, Spain

e-mail: agonzalezm@seom.org
}

patients who were believed to have localised disease prove to have a more advanced stage than anticipated. Studies on the surgical staging of apparent early-stage ovarian tumours suggest that up to $22-30 \%$ of patients who undergo a meticulous surgical staging procedure will ultimately have their disease upstaged. For this reason, specialisation is essential in the surgical treatment of this disease.

The outcome of adequately staged stage I patients is quite variable. There are patients with a low risk of relapse and an excellent prognosis showing a 5-year survival rate over $90 \%$. However, patients with adverse prognostic factors can reach a mortality rate at 5 years of $30-40 \%$. The identification of each group is critical, as long as the former group can be cured with surgery exclusively, but the second one warrants the administration of adjuvant chemotherapy. Grade, the rupture of the capsule and FIGO substage are the most potent and validated prognostic factors in order to separate both groups [5]. Patients with stage IA-B and grade 1-2 belong to the low-risk group. On the other hand, patients with stage IC-IIA, grade 3 or unfavourable histology like clear cells are classified in the high-risk group.

The value of adjuvant chemotherapy in stage I disease was definitively demonstrated in a combined analysis of two large European studies (ACTION and ICON). Although there was a wide range of stages (from IA to IIC) and grades ( 1 to 3 ) included in both studies, the analysis of 925 patients confirmed that the administration of adjuvant chemotherapy based on a platinum-salt is associated to an absolute benefit in disease-free survival at 5 years of $11 \%$ and an overall survival of $7 \%$, both values being statistically significant [6].

Nevertheless, since patients who underwent an adequate staging surgery (a third of the total) in the ACTION study did not benefit from adjuvant chemotherapy, doubts were raised as to the benefit of adjuvant therapy in this group of patients. Unfortunately, the number of patients was insufficient to obtain significant differences and the impact of staging surgery on the benefit obtained from chemotherapy in patients with optimal staging surgery is not completely clarified.

Another cornerstone study for the management of earlystage ovarian cancer was GOG-157 [7]. This trial compared the administration of 3 cycles of paclitaxel $\left(175 \mathrm{mg} / \mathrm{m}^{2}\right.$ over $3 \mathrm{~h}$ ) and carboplatin (AUC 7.5) vs. 6 cycles of the same 
combination in patients with stage I optimally staged. There were 457 patients recruited and $331(70 \%)$ met all eligibility criteria, the most frequent reason for ineligibility being incomplete surgical staging (107 patients). The risk of recurrence was 33\% lower for patients treated with 6 cycles. However, this difference did not reach statistical significance (95\% $\mathrm{CI}=0.49-1.16)$. Additionally, the 6-cycle arm was associated to a higher haematological toxicity and neurological grade $2-4$ toxicity ( $28 \%$ vs. $13 \%$ ). Based on this trial, the current standard chemotherapy in the adjuvant setting consists of at least 3 cycles of paclitaxel and carboplatin.

The analysis of the series of patients presented in this issue of Clinical and Translational Oncology can only be considered as a picture of the evolution in the treatment of ovarian cancer and a reflection of the normal practice in a European academic hospital. Ninety-eight patients received different platinum- and non-taxane-based chemotherapy before 1997, and from that time 60 additional patients received the combination of paclitaxel and carboplatin. Around $16 \%$ of patients had stage IIB or IIC, which are nowadays usually included in clinical trials for advanced disease. It is relatively surprising that a higher rate of grade 2 (47\%) and mucinous histology (33\%) was reported than in previous series. Regarding surgery, the rate of optimal surgery reported was $46.2 \%$, but interestingly this rate increased over time, from $35.7 \%$ before 1997 to $64.3 \%$ later, probably reflecting the acceptance of the absolute value of a comprehensive staging surgery in the most recent years, as has been adequately argued by the authors.

The rate of relapse reported in this trial is $25.9 \%$, which is very similar to the recurrence rate reported in the ACTION trial [6], the GOG-157 trial [7] and other European cohorts, like that presented by the GEICO Group (Grupo
Español de Investigación en Cáncer de Ovario) [8]. Taking into account that the median follow-up of this series is longer than 8 years, we can assume that this is a solid figure.

Can we be satisfied with this figure in our daily clinical practice? I would say not enough. Even considering that in this series around $16 \%$ of patients had stage IIB-IIC, still one in four patients have a relapse despite surgery and chemotherapy. However, this trial shows important real-life data that can help us in reaching some important conclusions for improving our practice. The first important message is that surgery is still the mainstay of treatment for early-stage ovarian cancer. Inadequate surgery can miss up to $30 \%$ of advanced stage; for this reason a rate of optimal surgery below $50 \%$ should be considered insufficient and we have to continue asking for specialised surgeons in ovarian cancer to perform staging surgery. The second important conclusion is that despite the administration of standard chemotherapy, there are a group of patients that are resistant and finally will develop a relapse. It is critical in the molecular and personalised medicine era to identify those patients that have a huge chance of relapse and to develop predictive factors of response to conventional chemotherapy or new agents. Moreover, if we were able to identify those patients that are going to relapse despite surgery and that are not going to be cured by adjuvant chemotherapy, we could develop new clinical trials with new agents for this high-risk population of early-stage patients. Unfortunately, early-stage ovarian cancer is a relatively uncommon disease and the possibility of launching large clinical trials is limited. For this reason, the effort of large cooperative groups and cooperation under the Gynecological Cancer Intergroup is the only way to answer this important question for our patients.

\section{References}

1. Jemal A, Siegel R, Xu J, Ward E (2010) Cancer statistics, 2010. CA Cancer J Clin 60:277-300

2. Hennessy BT, Coleman R, Markman M (2009) Ovarian cancer. Lancet 374:1371-1382

3. García-Saénz JA, Custodio A, Casado A et al (2011) Platinum-based adjuvant chemotherapy on moderate and high-risk stage I and II epithelian ovarian cancer patients. Long-term single institution experience and literature review. Clin Transl Oncol 13:121-132
4. González-Martín A, Toledo G, Chiva L (2010) Epithelial ovarian carcinoma: current evidences and future perspectives in the first-line setting. Clin Transl Oncol 12:418-430

5. Vergote I, De Brabanter J, Fyles A et al (2001) Prognostic importance of degree of differentiation and cyst rupture in stage I invasive epithelial ovarian cancer. Lancet 357:176-182

6. Trimbos JB, Parmar M, Vergote I et al (2003) International Collaborative Ovarian Neoplasm Trial 1 and Adjuvant Chemotherapy in Ovarian Neoplasm Trial: two parallel randomized phase III trials of adjuvant chemotherapy in patients with early-stage ovarian carcinoma. J Natl Cancer Inst 95:105-112

7. Bell J, Brady M, Lage J et al (2003) A randomized phase III trial of three versus six cycles of carboplatin and paclitaxel as adjuvant treatment in early stage ovarian epithelial carcinoma: a Gynecologic Oncology Group Study. Gynecol Oncol 88:156

8. Ruiz N, del Campo JM, Calvo E et al (2008) Early stages (I-IIa) epithelial ovarian cancer (EOC): preliminary data of the prospective register of the Spanish Group on Research of Ovarian Cancer (GEICO). Ann Oncol 19[Suppl 8]:viii211viii216. doi:10.1093/annonc/mdn512 